\title{
Design and Implementation of Fast Entry System for Equipment Downtime Information Based on .NET MVC Framework
}

\author{
Shicheng Hu, Yize Zhang \\ School of Economics and Management, Harbin Institute of Technology, Weihai 264209, China \\ zyz_hit@126.com
}

\begin{abstract}
In recent years, efficiency plays more and more important role in the work, improve enterprises' information entry efficiency has become a problem in front of the enterprise. Aiming at the process of enterprise downtime equipment information entry, this paper gives a detailed intruduction of the structure and fuction of the Fast Entry System for Equipment Downtime Information, as well as designing the comprehensive downtime information to improve the efficiency of entring. On the basis of enterprise needs, the system based on .NET MVC framework was designed and built on the visual studio 2010 development platform with SQL server 2008 express database in this paper. We describe the overall design of the system model, and explain how to use MVC framework in the system. This system has improved the entering process specification, correctness and efficiency greatly. That is to say, the system, having been put into practice in a manufacturing company in ShanDong province, saves time and labor to a great extent.
\end{abstract}

Index Terms - ASP.NET MVC, Data Modeling and Access, System of Information

\section{Introduction}

At the present, the domestic manufacturing industry are facing the fierce competition and as the core factors of restricting enterprise production efficiency, how to reduce the downtime equipment rate became more and more important in manufacturing enterprises. Although the domestic manufacturing are paying more and more attention to ERP system, but most of enterprise equipment downtime rate is calculated in manual mode. Even if some ERP system can achieve the collection of enterprise downtime equipment information, but for most companies, a particular production line, production workshop and its products are specific, so companies facing a problem that shutdown equipment information such as the basis of the production line, operation, equipment type are repeated entry, entrying information is error prone, large work volume on entry personnel and other issues.

In order to reduce the entry intensity of equipment downtime information, improve the efficiency and accuracy, this article make the usual repetitive equipment downtime information such as product line, process, equipment, product information and related constants data merge together to form a comprehensive downtime information. That is to say, recording multiple units only needs to choose a comprehensive downtime information and then sysytem will fill in the corresponding constants data. The Fast Entry System for Equipment Downtime Information based on .NET MVC platform can fully meet the requirements of equipment downtime information collection, bringing convenience for data entry personnel and improve the efficiency of the staff.

\section{Technology Applied In the System}

A. Introduction of $M V C$

ASP.NET MVC framework is a new develop pattern which is developed by Microsoft Company in April 2009 [1]. This is the first time that MVC development is thought fit in ASP.NET [2]. The MVC framework is designed to solve the problem that we can not separate View and Model in traditional ASP.NET development [3]. According to different function, the general application using MVC framework is divided into three parts which are Model, View and Controller [4].

The client layer: namely the V in the MVC (View), use the CSS and JavaScript technology implementation, as the showing and interfacing module of system function. In the B/S mode, the client through the browser to access.

WEB service layer: is the C (Controller) in MVC, use the .NET MVC framework for business control, responsible for interactions between data service layer and the client, calling the data service layer, and returning data model to the client layer for organizational performance [5].

Data service layer: is the M (Model) in MVC, on the basis of database, through the packaging of data Model to complete system.

\section{B. Introduction of SQL Server 2008}

This system uses SQL Server 2008 Express database management in Visual Studio 2010 (VS2010) to build data table [6]. We use ADO.NET Entity Data Model (EDM) in VS2010 to map related table in the database. And after that, we can access data in application.

With the Entity Data Model Tools, we can create a conceptual model from an existing database and then graphically visualize and edit our conceptual model. The ADO.NET Entity Data Model Designer allows us to create and modify entities, associations, mappings, and inheritance relationships. It can also generate C\# object-layer code.

\section{System Design}

\section{A. Functional Module}

According to actual needs, The Fast Entry System for Equipment Downtime Information mainly consists of five modules, each module holding corresponding sub-modules, 
which is shown in figure 1 . Next, we will introduce each module briefly.

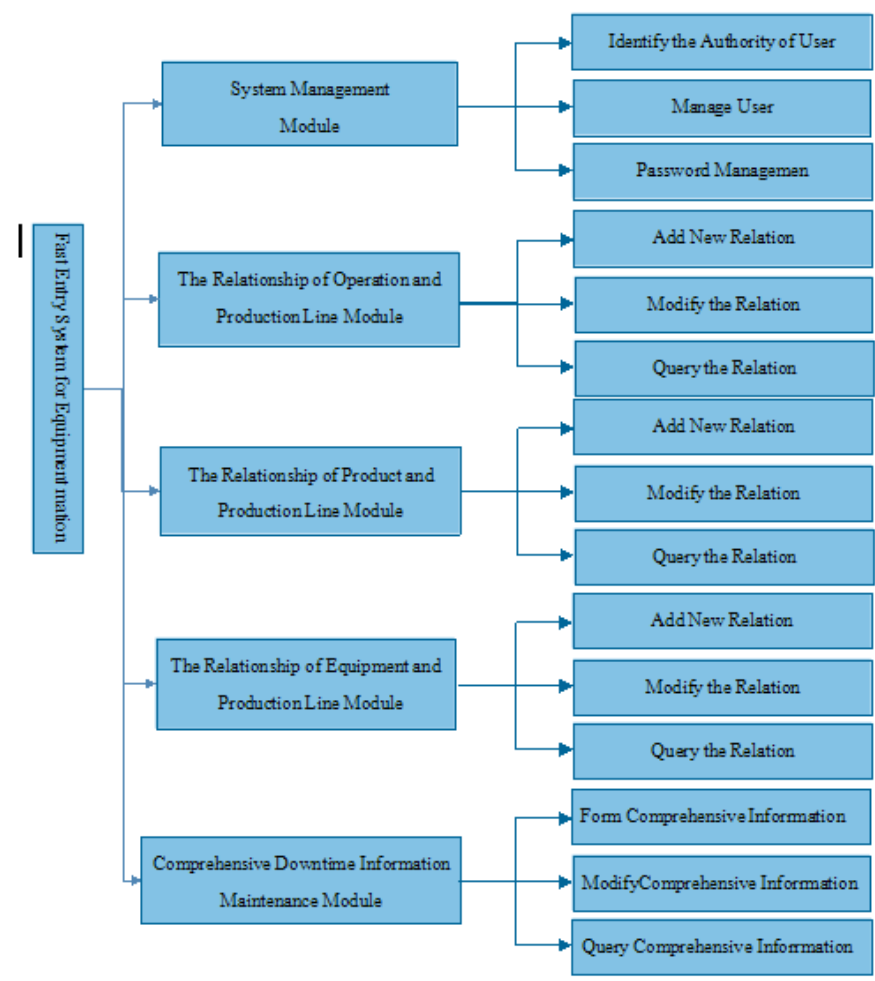

Fig. 1 Fast Entry System for Equipment Downtime Information functional module.

1) System Management Module: Manager identify the authority of users. The system shall provide individuation service and operation according to different people.That is to say, different users with different permissions on the downtime information.

2) The Relationship of Operation and Production Line Module: It mainly consists of production line information management and operation information management. It is convenient for users to modify relationship of operation and production line.

3) The Relationship of Product and Production Line Module: It mainly consists of production line information management and product information management. It is convenient for users to modify relationship of product and production line.

4) The Relationship of Equipment and Production Line Module: It mainly consists of production line information management and equipment information management. It is convenient for users to modify relationship of equipment and production line.

5) Comprehensive Downtime Information Maintenance Module: The maintenance is convenient for user set up the production line information, operation, equipment, product and other const data together, thus choose a comprehensive downtime information can fill the corresponding production line information, operation information, equipment information, product information automaticly. This module including the comprehensive information of add, modify, query functions.

System based on the production line, binding production line with the product, operation, equipment, and through setting the entry personnel permissions on production line, thus entry personnel with different permissions can input different information of downtime. In the comprehensive downtime information function, user combine authorized product, operation, equipment with production line to form a comprehensive downtime information.

\section{B. Relationship among Model, View and Controller}

According to system function design, in accordance with the MVC pattern to build software structure of the system as shown in figure 2.

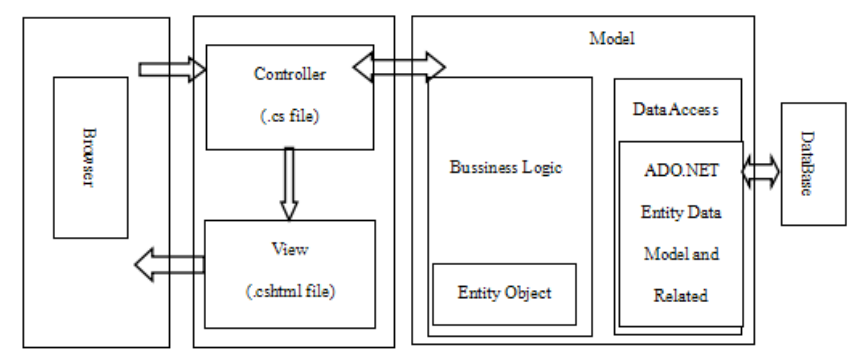

Fig. 2 Software Structure of The System.

Model is responsible for database data processing. In The Fast Entry System for Equipment Downtime Information, Model is used to manage the application behavior and data. It is divided into business logic section and data access section.

View is responsible for display the contents of the user interface. In other words, it is used to display model data in the user interface. For example, in the system, downtime information interface displays the detailed data which comes from the database; we call the interface a view. In General, the view can be a HTML page, but this system uses MVC2.0 razor view engine in VS2010 to complete the content display.

Controller is used to process the user's input or interactive commands, change model status, and select appropriate view to display the corresponding model data through controller. It is like a bridge linking views and models. In the system, when user sends request to input downtime information, controller will accept and deal with the request, and then put the related data into downtime information models. The existence of controller realizes the separation with view and business logic.

\section{Database Implementation}

Through the above analysis, entities of The Fast Entry System for Equipment Downtime Information are: production line, operation, equipment, products, user, comprehensive downtime information. Through further analysis, relationship between each entity as follows.

The connection of product line and operation: a product line generally contain a variety of operation, one operation can only belong to a production line, therefore, the contact is $1: \mathrm{n}$. 
The connection of production line and products: one production line can produce a variety of products, one product can be produced by production lines, as a result, the connection is $\mathrm{m}$ : $\mathrm{n}$.

The connection of production line and workshop: one production line can contain more than one workshop, a workshop can only belong to the whole production line, therefore, the contact is $1: \mathrm{n}$.

In conclusion, the database model can be expressed in figure 3.

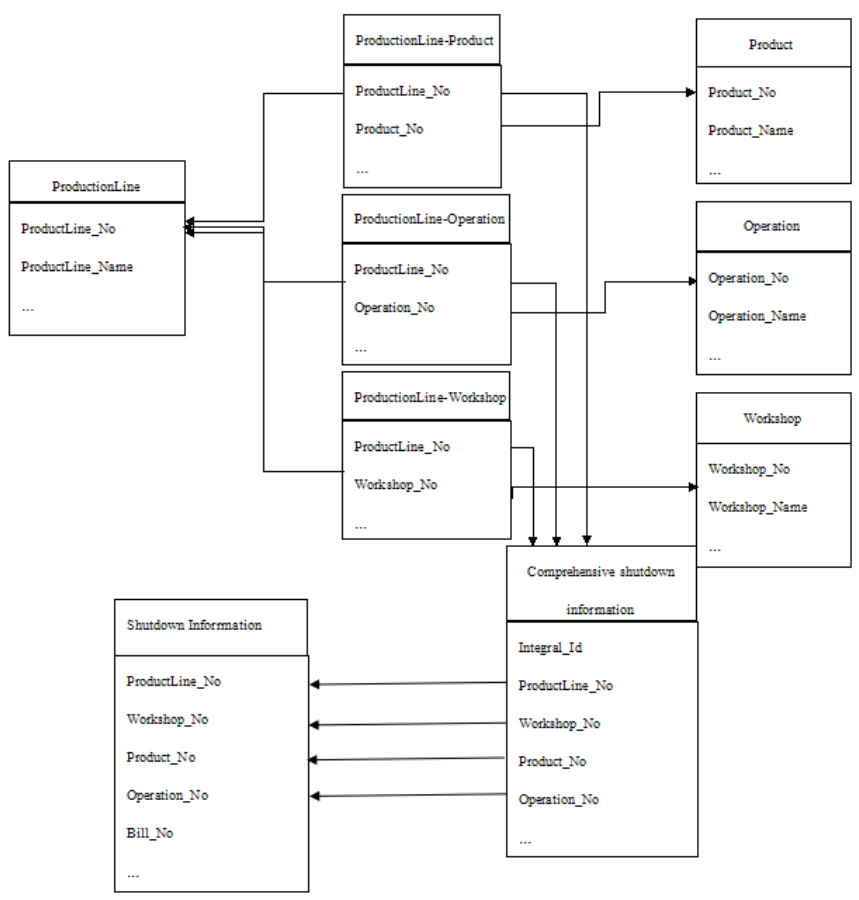

Fig. 3 The design of ADO.NET Entity Data Model.

\section{System Implementation}

\section{A. Comprehensive Downtime Information}

Comprehensive downtime information containing the information of production line, operation, equipment and product and other information of the downtime equipment. Production line as the connection of comprehensive downtime information, binding user and the operation, equipment serial number, product information, so as to user with different permissions can set different comprehensive downtime information. The relation of user and comprehensive downtime information is shown in figure 4 .

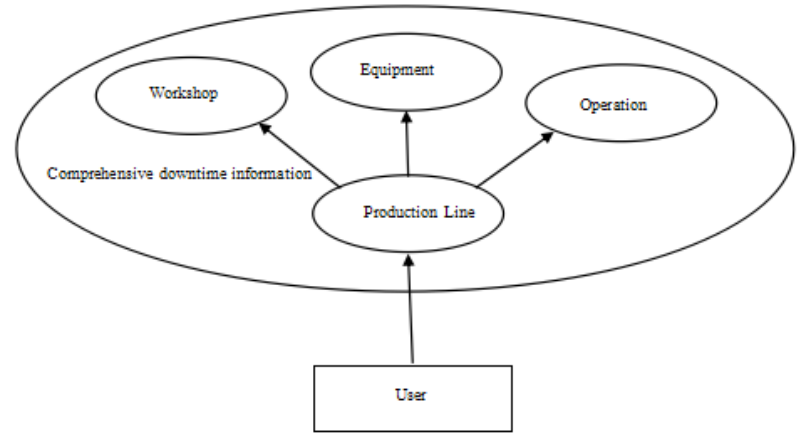

Fig. 4 The relation of user and comprehensive downtime informationl.

Comprehensive downtime information setup steps.

1) User login system, set up commonly used comprehensive downtime information, including: set up the production line and related products; Set up production line and operation related; Set up production line and equipment. The number and types of downtime information in the setting view belong to the permission of user.

2) As inputing the downtime information, according to the actual situation to choose suitable comprehensive downtime information, production line, workshop and other information can automatically fill in.

The examples of seting comprehensive downtime are presented from in figure 5.

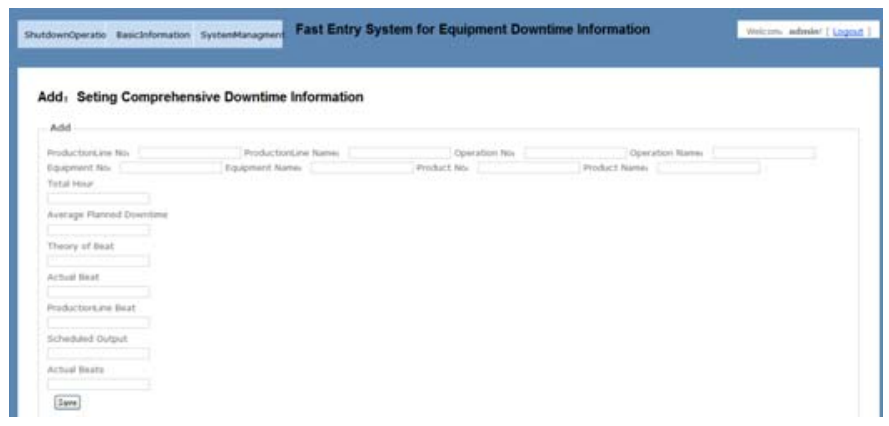

Fig. 5 Setup comprehensive downtime information page.

\section{B. Downtime Information}

Downtime information input is to enter all information of the downtime equipment into the system. In order to improve the efficiency of input, all the basic information of the downtime equipment such as production line code and name, product code and name, equipment number and name and all working hours and other constants can be filled automaticly by selecting comprehensive downtime information dropdown list. Downtime information input interface as shown in figure 6 . 


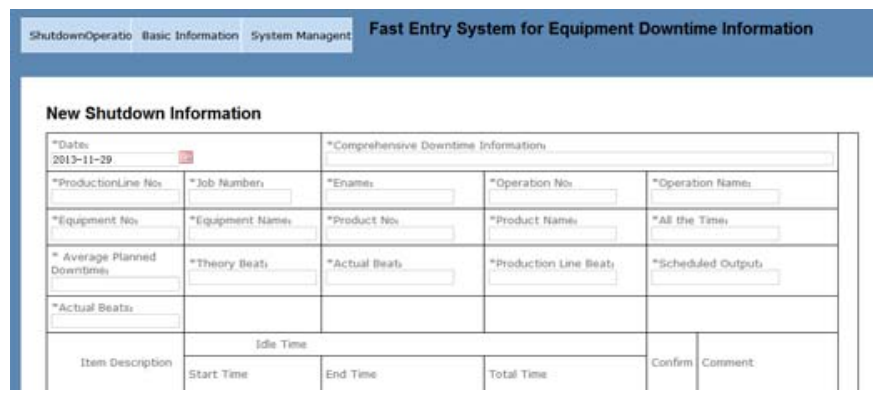

Fig. 6 Downtime information input page.

\section{Conclusion}

In order to meet the need of convenient management and quick ertry downtime equipment information, we use browser/server structure to design and implement Fast Entry System for Equipment downtime Information based on ASP.NET MVC framework. The interfaces in our system are clean and brief. Page respond is rapid. And data operation is convenient and fast. The actual operation has proved that our system is stable, efficient and safe. But in the actual entry work, the ERP project did not design to open, data cannot achieve seamless. How to realize the system integration, data seamlessly and intelligent diagnosis of idle equipment, automatic computing equipment efficiency and other functions on the existing enterprise downtime system remains to be further study.

\section{References}

[1] Zhi Li, Kebin Jia, Zhenzhen Li, Meng Yao, "Design and Implementation of Jewelry Selling System Based on .NET MVC Framework,” Int. Conf. Comput. Sci. Serv. Syst, CSSS, pp. 1575-1578, August 13, 2012.

[2] Hao Chibing. Asp.net 3.5 MVC framework and implement. Electronic Industry Press.2009.

[3] Lei Gao. An Intensive MVC Design Pattern Based on ASP.NET. Computer Engineering and Applications, 2010, (1):679-682.

[4] LI Hai-liang BAO Han-zong. Design and Development of Invoicing System Based on ASP.net MVC. Journal of Dongguan University of Technology. 2011, 18(1):37-40.

[5] JXIONG Jian-fang, GAO Ji, REN He-yu. Analysis and Study of ADO and ADO.NET Based on ASP.NET. Computer and Modernization, 2006, 7:36-38.

[6] Li Xiaohang. Application of LINQ Technology in the ASP.NET Programming. Science Mosaic, 2010, 8:33-35. 